\title{
(In) Visibilidade da Escola na Discussão Sobre o Uso Racional de Medicamentos
}

\author{
Nelson Machado do Carmo Júnior ${ }^{1}$ \\ Jackson Ronie Sá da Silva²
}

\begin{abstract}
Resumo:
A Organização Mundial de Saúde informa que o uso racional de medicamentos é uma prática a ser divulgada porque inúmeros agravos à saúde ocorrem em razão do abusivo consumo de medicamentos. Este artigo apresenta uma reflexão teórica sobre o papel da escola na divulgação de conhecimentos sobre os medicamentos e seu uso racional. Realizou-se uma pesquisa bibliográfica em bases de dados indexadas: Scielo, Lilacs, Medline e Google Acadêmico bem como em documentos oficiais do Ministério da Saúde e da Agência Nacional de Vigilância Sanitária do Brasil. Foram analisados 23 artigos, os quais deram origem a duas categorias de análise: "Invisibilidade da escola na discussão sobre o uso racional de medicamentos" e "Visibilidade da escola na discussão sobre o uso racional de medicamentos". 0 tema do uso racional de medicamentos não é abordado como deveria ser nas escolas da educação básica no Brasil. Quando abordado, apresenta-se de forma superficial a partir dos preceitos do modelo curativista de saúde. É necessário que a discussão da prática do uso racional de medicamentos esteja sendo feita nas escolas, tornando-se, desta forma, uma possibilidade cidadã de promoção da saúde.
\end{abstract}

Palavras-chave: Pesquisa bibliográfica. Uso racional de medicamentos. Educação em Saúde. Escola.

${ }^{1}$ Universidade Federal de Minas Gerais (UFMG). Hospital Risoleta Tolentino Neves. nelsonjuniorslz@hotmail.com

${ }^{2}$ Universidade Estadual do Maranhão (Uema). Centro de Educação, Ciências Exatas e Naturais. Departamento de Química e Biologia. prof.jacksonronie.uema@gmail.com 
(IN) SCHOOL VISIBILITY IN THE DISCUSSION ON THE RATIONAL USE OF MEDICINES

\begin{abstract}
:
The World Health Organization reports that the rational use of medicines is a practice to be publicized because numerous health problems occur due to abusive consumption of medicines. This article presents a theoretical reflection about the role of the school in the dissemination of knowledge about medicines and their rational use. A bibliographic search was carried out in indexed databases: Scielo, Lilacs, Medline and Google Academic as well as in official documents of the Ministry of Health and the National Sanitary Surveillance Agency. Twenty-three articles were analyzed which gave rise to two categories: "Invisibility of the school in the discussion about the rational use of medicines" and "Visibility of the school in the discussion about the rational use of medicines". The topic of rational use of medicines is not approached as it should be in primary schools in Brazil. When approached, it presents itself superficially from the precepts of the curativist model of health. It is necessary that the practice of rational use of medicines in schools, becoming a tool for health promotion.
\end{abstract}

Keywords: Bibliographic Research. Rational Use of Medications. Health Education.School. 
De acordo com a Organização Mundial de Saúde (OMS), o uso racional de medicamentos é uma prática que precisa ser divulgada porque inúmeros agravos à saúde da população (e em especial o público infanto-juvenil) ocorrem em grande escala em razão do abusivo consumo de medicamentos. Além disso, faz-se necessário promover a cultura de receitar corretamente os medicamentos bem como os sujeitos sociais terem acesso às informações médicas e farmacêuticas divulgadas por profissionais da saúde, como médicos, farmacêuticos, enfermeiros ou, numa perspectiva mais abrangente, serem informados por profissionais da educação (professores) que, mediante aulas contextualizadas e problematizadoras, discutam sobre os aspectos preventivos do processo saúde-doença (ORGANIZAÇÃO..., 2010).

Essa preocupação sobre o uso racional de medicamentos é válida e necessária pelas situações que envolvem a segurança de adolescentes ao fazerem uso de diversas classes de medicamentos no ambiente escolar, sendo esta prática caracterizada como automedicação (ABRAHÃO; GODOY; HALPERN, 2013; DAL PIZZOL et al., 2006). Catalán (2009) aponta a importância de tratar a promoção da Educação em Saúde de forma transversal e assegurar ações que transcendam a sala de aula e a escola, tentando transformá-la num centro promotor de saúde na comunidade em que estiver inserida.

A escola é uma instituição que tem passado por importantes transformações sociais e, por intermédio da cultura escolar, promovido a produção de inúmeros saberes que vão influenciando as culturas a partir do que é produzido na Ciência e Tecnologia, no trabalho e em outros espaços de interação humana (BRASIL, 2013a).

A cultura do consumo de medicamentos é algo que tem chamado a atenção de organismos nacionais e internacionais do setor Saúde. A Educação, enquanto campo que lida com o discurso do ensino e da aprendizagem, constantemente é requisitada a contribuir na discussão do uso indiscriminado e prejudicial de medicamentos (BRASIL, 2007; ORGANIZAÇÃO..., 2002; BRASIL, 1997).

A automedicação tem sido uma prática recorrente entre alunos de escolas de diversas regiões do país (ABRAHÃO; GODOY; HALPERN, 2013; CORRÊA et al., 2013; COSTA et al., 2011; SILVA et al., 2011). De acordo com Vitor et al. 
(2008), a automedicação é caracterizada pelo uso de medicamentos sem a prescrição de um profissional médico, quando o usuário decide qual medicamento utilizar, geralmente aconselhado por pessoas não habilitadas. É necessário que as instituições sanitárias fiquem atentas, principalmente pelos casos de intoxicação nessa parcela da população (BOCHNER, 2006).

Segundo dados do Sistema Nacional de Informações Tóxico-Farmacológicas (Sinitox), a maior percentagem de intoxicação é por medicamento, correspondendo a 28,45\%. Desse total, a faixa etária mais atingida é a de crianças menores de cinco anos, que representam 30,8\% dos casos. Os medicamentos lideram os casos de intoxicação no Brasil entre jovens de 15 e 19 anos, correspondendo a 8,6\% no ano de 2013 (BRASIL, 2013b).

Medicamentos estão no imaginário social e simbolizam a possibilidade de resolução dos problemas de saúde. Discursos sobre terapêutica, cura e prevenção estão associados ao consumo de medicamentos. Desta forma, artefatos culturais, ${ }^{3}$ como mídia, cinema, televisão, livros, revistas, dentre outros, veiculam aquilo que a população deseja ouvir: comprimidos, xaropes, cápsulas, injetáveis, cremes, pomadas, etc., são objetos terapêuticos para a cura de males orgânicos e psicológicos. Quem impulsiona a disseminação dessas informações? A indústria farmacêutica.

A indústria farmacêutica se sustenta por meio de discursos que simbolizam o sentido oposto do que institui a saúde coletiva. O que pretende a indústria farmacêutica? Produzir e vender medicamentos sem ter a preocupação de avaliar os riscos do consumo de princípios ativos farmacêuticos em larga escala, aumentar o capital em detrimento das demandas de enfermidades da população, estimular o consumo de medicamentos utilizando-se da publicidade e propaganda (MACHADO; LESSA, 2012; ANGELL, 2009; AQUINO, 2008; NASCIMENTO, 2005).

\footnotetext{
${ }^{3}$ É possível afirmar que "a educação se dá em diferentes espaços do mundo contemporâneo, sendo a escola apenas um deles, ou seja, somos educados por imagens, filmes, textos escritos, pela propaganda, pelas charges, jornais e principalmente a televisão" (COSTA; SILVEIRA; SOMMER, 2003, p. 22).
} 
Paradoxalmente, a saúde coletiva nos alerta para os excessos da indústria farmacêutica. Em linhas gerais, quais são os pressupostos da saúde coletiva quando o tema é o consumo de medicamentos? Ela visibiliza e divulga informações sobre práticas globais de saúde para promover o bem-estar da população, tendo como pilar a igualdade de oportunidades ao acesso responsável aos medicamentos e se preocupa com o tratamento, a cura e a prevenção de processos patológicos que maltratam os sujeitos sociais (MACHADO; LESSA, 2012; ANGELL, 2009; AQUINO, 2008; NASCIMENTO, 2005).

A escola precisa ser percebida como um espaço de diálogo quanto aos temas da educação em saúde, do uso racional de medicamentos e da automedicação (CARMO-JÚNIOR, 2013). Saúde é uma condição complexa e seu conceito vai além da ideia de "ausência de doença". É importante que se pense (e seja divulgado pela escola, por exemplo) um conceito ampliado de saúde: uma condição que envolve determinantes biológicos, sociais, econômicos, políticos, culturais e educacionais (CARMO-JÚNIOR, 2013; CÂMARA et al., 2012; LAURELL, 1982).

Neste artigo apresentamos uma reflexão teórica sobre o papel da escola e dos professores na divulgação de conhecimentos sobre os medicamentos e seu uso racional. Pretendemos, também, identificar e problematizar os discursos educativos que sinalizam para a importância da educação em saúde escolar no empoderamento de práticas educativas para a minimização dos problemas sociais advindos do uso inadequado de medicamentos.

\section{Procedimentos Metodológicos}

No período de outubro de 2013 a janeiro de 2014, realizamos uma pesquisa bibliográfica e análise documental acerca da temática da Educação em Saúde e do uso racional de medicamentos (SÁ-SILVA; ALMEIDA; GUINDANI, 2009). Foram selecionadas publicações indexadas nas bases de dados Scielo, Lilacs, Medline e Google Acadêmico, bem como a busca por artigos e documentos oficiais nas bases de dados do Ministério da Saúde do Brasil (MS) e da Agência Nacional de Vigilância Sanitária (Anvisa), as quais forneceram 
importantes informações. Foram adotados os seguintes critérios de inclusão para a seleção de artigos e documentos oficiais: artigos completos (original, reflexão, relato de experiência, atualização e revisão de literatura) e artigos publicados em português independente da data da publicação. Ainda, como critério de inclusão: os artigos e documentos deveriam conter em seu título e/ou resumo os seguintes descritores: "uso de medicamentos", "uso racional de medicamentos", “escola”, "professores", "educação em saúde”, "automedicação”, “consumo de medicamentos", “adolescentes", “escolares", “estudantes", “alunos”. Foram excluídos os artigos que não estavam condizentes com as temáticas do uso racional de medicamentos e da Educação em Saúde. Outro critério de exclusão: não analisar artigos de literatura estrangeira (para conhecermos a realidade nacional a respeito do tema).

Após a catalogação dos materiais bibliográficos, o passo seguinte foi a seleção dos referenciais teóricos que iriam compor a análise documental: 23 textos compuseram o corpus analítico. Os referenciais bibliográficos analisados passaram pelo processo de categorização após leitura flutuante e leitura em profundidade (MINAYO, 2013; SÁ-SILVA; ALMEIDA; GUIDANI, 2009). As categorias de análise construídas foram: "Invisibilidade da escola na discussão sobre o uso racional de medicamentos" e "Visibilidade da escola na discussão sobre o uso racional de medicamentos" (Anexo).

\section{Invisibilidade da Escola na Discussão sobre o Uso Racional de Medicamentos}

Quem deve informar sobre o uso adequado de medicamentos ou quais sujeitos são autorizados a falar da automedicação e do uso incorreto de medicamentos? Nas publicações analisadas esta questão é recorrente. Encontra-se na literatura referência aos profissionais responsáveis por esclarecer sobre o uso de medicamentos à população. São listados médicos e farmacêuticos (BRASIL, 2015; FOELLMER; OLIVEIRA; MOREIRA, 2010). Não identificamos, entretanto, qualquer referência ao professor e à escola como sujeitos e instituição que podem ser capazes de contribuir nessa importante discussão. Alguns documentos 
deixam evidente os profissionais que devem assumir a função de divulgadores das informações sobre os medicamentos. Farmacêuticos são os profissionais de eleição para promoverem a educação em saúde na prevenção ao uso incorreto de medicamentos:

[...] entende-se que o farmacêutico é o profissional da saúde que convive com este tipo de problema (BARTA; OLIVEIRA, 2010, p. 189).

[...] a população deve ser sensibilizada no sentido de diminuir as práticas inadequadas que conduzem ao uso irracional de medicamentos e [...] ser norteada a recorrer ao farmacêutico para monitorização e orientação sobre uso de medicamentos (ORGANIZAÇÃO PAN-AMERICANA DE SAÚDE), 2007, p. 19-20).

Existe unanimidade no discurso das 23 publicações: o uso incorreto de medicamentos constitui prática que deve ser abandonada. E mais: a população precisa conhecer os benefícios e riscos dos medicamentos. Além do farmacêutico - conhecido como "profissional do medicamento"- existem outros sujeitos que lidam com medicamentos diariamente. Os médicos foram citados, mas farmacêuticos e médicos não dão conta dessa complexa ação. Deve-se ampliar o espectro de sujeitos e locais para a divulgação das informações.

[...] Médicos, farmacêuticos, laboratórios, dirigentes, cidadãos, ONGs e o governo em todas as suas esferas devem se unir para encontrar a melhor maneira de disponibilizar informação qualificada e isenta que permita a cada um cumprir com suas responsabilidades dentro de uma política de uso racional de medicamentos (OLIVA, 2007, p. 2).

O papel do farmacêutico foi investigado na propagação de informações sobre o uso correto e responsável de medicamentos. O profissional médico foi o mais citado com $36,1 \%$ e o farmacêutico representou $18,1 \%$ como local de obtenção de informações sobre os medicamentos (SILVA et al., 2009, p. 101). [...] sendo o profissional médico a principal fonte de informação aos adolescentes (SILVA et al., 2009, p. 102).

Algumas instituições são qualificadas como espaços adequados para falar sobre o uso incorreto e abusivo de medicamentos: laboratórios farmacêuticos, pessoas comuns, gestores da área do medicamento, organizações e associações 
(de bairro, profissional), sindicatos, etc. Todos devem estar empenhados para “disponibilizar informação adequada" (OLIVA, 2007, p. 2). Esta percepção aponta para a possibilidade de os laboratórios farmacêuticos serem parte consciente desse processo de informação qualificada. É importante lembrar, no entanto, que a indústria farmacêutica visibiliza um discurso de consumo pelo consumo e sua perspectiva é potencializar o lucro mediante o uso de medicamentos pela população em grande escala.

Seria possível a indústria farmacêutica apresentar informações sobre o uso de medicamentos de forma a valorizar uma educação para o cuidado com os riscos? A indústria e o varejo farmacêuticos se utilizam de todos os artefatos midiáticos e espaços sociais para divulgar informações sobre medicamentos, mas numa perspectiva comercial. Essas informações, todavia, não são isentas de qualquer imparcialidade no discurso, conforme apontado por Angell (2009). $\mathrm{Na}$ verdade, o que predomina é a parcialidade do marketing, da venda e do consumo desenfreado.

Nas publicações analisadas tiveram destaque as drogarias e farmácias, a família, ambientes sociais, além da propaganda, como possíveis locais de divulgação de informações acerca dos medicamentos.

[...] a população recorre à farmácia como substituto dos serviços de saúde e do médico, aceitando, portanto, indicação de atendentes sem se preocuparem com o fato de que eles não são as pessoas mais indicadas para tal função (BARROS, 1997, p. 122-123).

Considerando os riscos da automedicação que estão diretamente associados a má qualidade da oferta dos medicamentos e dos serviços de saúde, incluindo o serviço prestado nas farmácias e drogarias (LEITE; VIEIRA; VEBER, 2008, p. 797).

Fatores como a disponibilidade de medicamentos na farmácia doméstica, a facilidade de aquisição de medicamentos em farmácias e drogarias, bem como a sua utilização para contornar situações sintomatológicas da dor são resultantes da propaganda massiva e da indicação de familiares e amigos (SILVA et al., 2009, p. 102). 
[...] o uso abusivo de medicamentos por crianças e adolescentes pode ser agravado, no Brasil, pela facilidade de acesso a esses produtos, tendo em vista o número elevado de farmácias e drogarias[...] (DAL PIZZOL et al., 12006, 114).

Os documentos categorizados fazem referência aos locais possíveis onde os discursos sobre o uso de medicamentos são realizados. Nenhum dos documentos incluídos nesta categoria relata a escola como um provável local de problematização dos discursos envolvendo informações sobre o uso de medicamentos, invisibilizando-a. Vejamos outros trabalhos que apontam diferentes locais para se falar sobre o uso de medicamentos:

[...] a influência do ambiente familiar sobre o uso de medicações, seja por estímulo direto junto aos adolescentes, seja pelo exemplo (SILVA; GIUGLIANE, 2004, p. 331).

[...] em propagandas que direcionam o público e geral a sítios na internet ou que induzem o consumidor a pedir informações do tipo "pergunte ao seu médico" (PALACIOS; REGO; LINO, 2008, p. 895).

Na população brasileira, a automedicação é prática comum, e, naquela faixa etária, previamente à entrada na fase adulta, os indivíduos ficam expostos ao uso de medicamentos pela família e pela mídia (ALMEIDA et al., 2012, p. 217).

Podemos perceber que os discursos sobre os medicamentos remetem às expressões "de pouca gravidade", "aos sintomas corriqueiros" ou "passageiros" que aparentemente são solucionados nos ambientes sociais, conforme aponta Nascimento (2005, p. 182). Nesse discurso está presente a lógica do incentivo ao consumo, da automedicação e da medicalização como cultura (CARVALHO et al., 2015; MACHADO; LESSA, 2012; FOUCALT, 2010; NASCIMENTO, 2005).

As farmácias e drogarias, o ambiente familiar e os variados locais onde se realizam as propagandas de medicamentos, são espaços em que a prática da automedicação é disseminada. As publicações analisadas nos mostram isso. Também identificamos que crianças e adolescentes, ou seja, indivíduos em idade escolar são as faixas etárias ou o público afetado diretamente com a 
automedicação, o uso irracional e a intoxicação por medicamentos (BRASIL, 2013b), seja pela facilidade no acesso, por exemplo, dentro de casa, seja por estar exposto à mídia. Ainda aparece como elemento importante o uso do medicamento associado a contextos sociais e culturais, como usar determinado medicamento para ser aceito em determinado grupo social.

[...] Neste período da vida, estão suscetíveis à influência do grupo ("ser aceito"), o que pode incentivar o uso de medicamentos por razões estéticas, como aqueles usados para emagrecer ou ganhar massa muscular (ALMEIDA et al., 2012, p. 217).

É importante voltarmos a questionar se a escola não poderia realizar essa interlocução entre a necessidade de usar o medicamento e o pensamento crítico sobre a quantidade de informações veiculadas aos adolescentes acerca desse uso. Porque não incluir a escola como espaço de discussão de tão relevante tema? Ressaltamos que o ambiente escolar é um espaço de empoderamento e problematização e os professores são sujeitos da construção de uma visão crítica a respeito de variados temas em saúde coletiva, como o uso de medicamentos. As instituições e sujeitos listados até o momento simbolizam o incentivo ao consumo e à automedicação. A escola e os professores, no entanto, não podem cumprir esse papel de incentivo à automedicação. Eles atuam de forma crítica, promovendo saúde no ambiente escolar.

Identificamos, em algumas publicações, uma contradição: existe um sujeito que não deve falar sobre o uso correto de medicamentos - o balconista. O balconista é literalmente descredenciado a se posicionar. A fala do balconista representa a voz mais evidente da venda e simboliza a representação do lucro pelo lucro:

[...] os balconistas atuam, erroneamente, como prescritores, agindo favoravelmente ao uso inadequado dos medicamentos. [...] a população recorre à farmácia como substituto dos serviços de saúde e do médico, aceitando a indicação de atendentes sem se preocuparem com o fato de que eles não são as pessoas mais indicadas para tal função (BARROS, 1997, p. 123-124). 
[...] prescrição ou indicação de medicamentos por pessoas não habilitadas, como amigos, familiares e mesmo balconistas de farmácia, caracterizando exercício ilegal da medicina (KOVACS; BRITO, 2006, p. 336).

Na farmácia, ensino e aprendizagem ocorrem de forma espontânea, sem que os próprios participantes do processo tenham consciência das consequências, porém é uma prática consciente (oferecer o medicamento) por pessoas instaladas dentro das farmácias sem o conhecimento necessário, tornando um discurso educacional duvidoso e perigoso.

As pessoas acabam por receber informações de vários lados: dos profissionais médicos e de saúde, dos balconistas e também da própria mídia que, por meio de um processo educacional irresponsável, gera consumidores da lógica dos "sintomas menores", dos “sintomas toleráveis", daquilo que não precisa do médico ou farmacêutico para intervir ou, até mesmo, da escola para conscientizar. Nesse sentido, a automedicação dita responsável acaba por assumir uma conotação na disputa de poder e não na lógica da mudança na intervenção e na promoção da saúde e atenção primária.

Esse processo educacional (que acontece fora da escola) fundamenta-se na imposição do consumo de medicamentos sobre a população, porque é uma prática que não expõe a dialógica, mas sim uma marginalização que visa a doutrinar aqueles que não detêm o conhecimento; essa é uma prática alienante que é assistida e reproduzida dentro de um sistema que não visa uma educação libertadora e transformadora. Pelo contrário, reafirma conscientemente modelos hegemônicos da sociedade capitalista.

Ou seja, temos de destacar, então, que as produções científicas não podem esquecer que a escola é um espaço social que pode dar uma importante contribuição ao campo da saúde pública na minimização dos problemas gerados pelo uso incorreto de medicamentos. A escola pode questionar hábitos desfavoráveis, como a automedicação, o incentivo ao consumo de medicamentos e até a própria 
medicalização da educação. ${ }^{4} \mathrm{O}$ s professores são sujeitos que podem contribuir. $\mathrm{O}$ médico, o farmacêutico e o balconista, no entanto, foram os profissionais elencados para falar sobre os medicamentos, de acordo com os materiais analisados.

A família também reforça a automedicação. A mídia e a indústria farmacêutica estimulam essa prática. A escola não é lembrada como possibilidade para o exercício da Educação em Saúde na prevenção ao uso incorreto de medicamentos. A escola e os professores podem contribuir para que se faça o caminho inverso da indústria farmacêutica, com lógica prioritariamente capitalista das farmácias que não valorizam a presença do farmacêutico e que incentivam a ação de balconistas motivados pela venda competitiva e em larga escala.

\section{Visibilidade da Escola na Discussão Sobre o Uso Racional de Medicamentos}

A escola tem sua parcela de contribuição quando nos referimos ao tema da promoção da saúde, além de influenciar de forma positiva na comunidade escolar (COSTA, et al., 2013; FERRARI DE LIMA; MALACAME; STRIEDER, 2012; MOTA, 2011; GOMES; HORTA, 2010; CARDOSO; REIS; IERVOLINO, 2008).O processo de transformação pelo qual passa a escola é fundamental para que se aprofunde a necessidade de esse local incorporar os discursos e interlocuções sobre o tema promoção da saúde e também do uso de medicamentos.

A escola é um importante espaço para o encontro entre a saúde e a educação, possibilitando diversas iniciativas de promoção da saúde, incluindo o tema do uso racional de medicamentos dentro dessas iniciativas (CASEMIRO; FONSECA; SECO, 2014; FOELLMER; OLIVEIRA; MOREIRA, 2010).

\footnotetext{
${ }^{4}$ Fenômeno associado à cultura de medicalizar crianças e adolescentes com diagnóstico de TDAH em idade escolar, principalmente relacionado a comportamentos excessivos, transtornos de aprendizagem e/ou outras dificuldades no ambiente escolar. Essa cultura deixa de lado as questões de ordem política, social, educacional, linguística, afetiva e as transforma em aspectos de ordem biológica (SIGNOR; BERBERINA; SANTANA, 2016).
} 
A articulação entre a promoção da saúde e a educação escolar são duas potenciais ferramentas para instituição de uma nova cultura na escola, seja ela de forma integrada, transversal e intersetorial. Isso proporciona maior diálogo entre os serviços de saúde, a comunidade e demais setores da sociedade (BRASIL, 2006). É importante ressaltar que as ações educativas se configuram como ações preventivas e de conscientização e têm uma potencial vantagem em relação às ações curativistas. Nesse sentido, é preciso que se valorize a relação professor/escola/profissional de saúde e se estabeleça vínculos que atendam às necessidades e demandas da comunidade, além de a escola poder criar seus próprios projetos de promoção da saúde. É preciso abandonar o antigo modelo educacional e ter a escola como um ponto de convergência na promoção da saúde (COSTA et al., 2013; FERRARI DE LIMA; MALACAME; STRIEDER, 2012; ZANCUL; COSTA, 2012; GOMES; HORTA, 2010; COSTA; SILVA; DINIZ, 2008; GONÇALVES, et al., 2008; SANTOS; BÓGUS, 2007).

A escola também pode desenvolver e executar práticas pedagógicas envolvendo a promoção da saúde e o uso de medicamentos em conteúdos formais com questões relacionadas à automedicação. Essa postura proporciona a formulação de ideias sobre os benefícios e malefícios que podem ser provocados pela prática da automedicação.

Dessa forma, podemos mostrar as relações interdisciplinares entre os conhecimentos científicos, fundamentais para a compreensão dos diversos assuntos da vida cotidiana (LAGO et al., 2016). Explorando os trechos dos documentos e artigos incluídos nessa categoria, percebemos um espaço de construção de uma discussão produtiva sobre o tema do uso de medicamentos e suas relações com o espaço escolar:

[...] a escola cumpre um papel destacado na formação dos cidadãos para a construção de hábitos saudáveis, na medida em que o grau de escolaridade e de desenvolvimento cognitivo contribuem para o nível de saúde da população, (BRASIL, 2007, p. 26).

O professor tem uma participação essencial como promotor de saúde. Durante as aulas, ele pode despertar nos alunos e na comunidade atitudes para a promoção da saúde (BRASIL, 2007, p. 27). 
A inserção de temas "Uso Racional de Medicamentos" e "a propaganda de medicamentos" no currículo das séries iniciais deve ser baseada em aspectos que: enfoquem a prevenção e o uso indevido de medicamentos [...] (BRASIL, 2007, p. 71).

Nossa análise parte do documento governamental que traz trechos importantes para o entendimento do papel da escola na construção do sujeito crítico, enfocando aspectos de prevenção relacionados ao uso dos medicamentos, da promoção da saúde e da construção de hábitos saudáveis individuais e coletivos. O documento elaborado por Brasil (2007) foi um esforço inicial para que os demais estudos encontrados na literatura pudessem problematizar a respeito do tema da Educação em Saúde e, mais especificamente, do uso de medicamentos e sua relação com a escola. Identificamos que os trechos desse documento abordam como a escola pode ser um espaço apropriado para a prática da educação em saúde e a participação do professor nesse processo. Esse primeiro movimento ainda apresenta concepções pautadas na lógica curativista que não abordam de forma clara como devem ser construídas e desenvolvidas tais ações na escola.

Alguns trabalhos fazem o contrário: visibilizam a participação da escola e dos professores como sujeitos centrais, além da contribuição que os mesmos podem proporcionar à temática do uso racional de medicamentos.

A escola, por ser um espaço de formação, onde se busca que o aluno desenvolva uma postura crítica e reflexiva em relação ao seu cotidiano, deve se preocupar com a influência dos meios de comunicação que tentam convencer, estimular o consumo de medicamento (SANTOS, 2008, p. 28).

[...] E esperamos ainda, que esses professores possam ajudar as crianças e os pais a entender melhor a importância do medicamento para saúde de todos (MANGUEIRA et al., 2011, p. 6).

Em se tratando da escola fundamental e, tendo em vista o papel que ela representa na formação do indivíduo e a sua potencialidade de estimular a criação de conceitos, assim como sua atitude questionadora e crítica em relação à realidade, objetivou-se investigar o conhecimento das práticas populares de saúde (SANTOS; DIAS; MARTINS, 1995, p. 221). 
Assumir essa postura crítica e reflexiva a respeito do tema demonstra que já existe uma preocupação na divulgação do papel da escola nas ações comunitárias em favor de uma discussão curricular sobre o processo saúde-doença relacionado ao uso de medicamentos pelos atores sociais (CASEMIRO; FONSECA; SECCO, 2014; FOELLMER; OLIVEIRA; MOREIRA, 2010).

Identificamos algumas limitações nos artigos e documentos analisados que apresentam o tema a partir de uma perspectiva escolar. Não há indicações didáticas de como fazer ou mesmo realizar a inserção desse tema. Isso é um fator limitante, pois o professor fica refém de análises e observações gerais, e sem apontamentos que solucionem a forma de abordagem da educação em saúde e das informações sobre medicamentos na escola.

Esse aspecto gera duas situações que merecem análise: o tema é problematizado de maneira equivocada e acaba por produzir compreensões confusas pelo fato de a abordagem não ser parte do currículo nas universidades e escolas da educação básica, principalmente na formação dos professores e na estruturação dos cursos de licenciatura.

Estudos sobre a percepção de professores de Ciências e Biologia têm demonstrado um conhecimento elementar sobre o tema da educação em saúde, pautado no discurso da enfermidade e da cura, falta de informação sobre como abordar tal tema, além da insuficiente formação acadêmica durante a Graduação (ZANCUL; COSTA, 2012; ZANCUL; GOMES, 2011).

Convencionaram-se os currículos dos cursos de Ciências Biológicas (Bacharelados e Licenciaturas) a tão pouco ou quase nada sobre a promoção da saúde. As Diretrizes Curriculares Nacionais abordam, de maneira tímida ou inicial, que o biólogo (bacharel ou licenciado) deve se formar e ser consciente da necessidade de atuar com qualidade e responsabilidade em prol das políticas de saúde (BRASIL, 2001). Trabalhos recentes, no entanto, apontam perspectivas de construção de práticas pedagógicas e ferramentas metodológicas dentro da escola que possam contribuir no desenvolvimento desse tema no Ensino Fundamental, estabelecendo discursos e enfrentamentos com instituições que representam a lógica do consumo (LAGO et al., 2016; RICHETTI; FILHO, 
2014; ANDRADE; SOUSA, 2013; SILVA; PINHEIRO, 2013). Vejamos alguns trechos que visibilizam o papel social da escola, que pode contribuir a respeito de informações sobre o uso de medicamentos.

[...] ao tratar o corpo humano e a saúde, é ter maior conhecimento do próprio corpo e percepção das necessidades biológicas, afetivas, sociais e culturais em todas as fases do desenvolvimento (DISTRITO FEDERAL, 2014, p. 114).

[...] Evidencia-se a contribuição para a formação de alunos de ensino fundamental que se tornaram mais esclarecidos quanto ao uso correto de medicamentos e outros temas ligados à saúde [...] (ABJAUDE et al., 2012, p. 273).

Aqui percebemos o discurso da escola como um ambiente de conscientização, de formação do pensamento e de construção social no campo da saúde coletiva e do uso correto de medicamentos. Estes discursos mostram a importância que a escola tem na abordagem do uso correto de medicamentos (CASEMIRO; FONSECA; SECCO, 2014; COSTA et al., 2013; FERRARI DE LIMA; MALACAME; STRIEDER, 2012; MOTA, 2011; FOELLMER; OLIVEIRA; MOREIRA, 2010; GOMES; HORTA, 2010; CARDOSO; REIS; IERVOLINO, 2008). Segue mais alguns discursos:

[...] A maioria dos riscos de morbidade relacionados a medicamentos poderia ser minimizado caso a cultura sobre o uso adequado de medicamentos estivesse presente na escola desde a infância (CORR $\hat{E}$ A et al., 2013, p. 3.073).

[...] Um destes ambientes pode ser a escola, pois a mesma é reconhecida como um espaço propício para tais atividades de promoção e prevenção, além de possuir uma responsabilidade social neste sentido (DANDOLINI et al., 2012, p. 1324).

[...] desenvolver, na escola pública, um trabalho conjunto com os profissionais da educação (ABJAUDE et al., 2012, p. 273).

[...] Trouxe, para dentro da sala, a realidade, em relação ao uso irracional de medicamentos, fez ênfase em discutir com eles e inclusive se colocar dentro do processo (SANTOS, 2008, p. 49)

As informações extraídas do material categorizado apontam para conceitos de uma educação crítica, além de importantes conceitos em saúde coletiva. Os artigos e documentos agrupados na categoria "invisibilidade da escola na 
discussão sobre o uso racional de medicamentos" abordam uma lógica diferente e não reconhecem o espaço escolar como um local para a discussão de informações sobre o uso correto de medicamentos. Já os trabalhos incluídos na categoria "visibilidade da escola na discussão sobre o uso racional de medicamentos", localizam o espaço escolar e os professores como sujeitos que podem contribuir na superação dos problemas relacionados ao uso de medicamentos.

A escola assume uma postura oposta ao da indústria farmacêutica e dos agentes da educação mercadológica pautada na empurroterapia. ${ }^{5} \mathrm{Ou}$ seja, assume uma postura com influências positivas ao uso racional de medicamentos. $\mathrm{O}$ professor tem destaque na construção do diálogo educacional sobre esse tema, pois pode despertar a atenção dos alunos e da comunidade escolar para o tema do uso racional de medicamentos.

Existe uma influência dos discursos estabelecidos entre a escola e os alunos auxiliando estes a refletir sobre o uso de medicamentos e a preservação da própria saúde, inclusive colocando-os dentro do processo de construção de uma ação pedagógica transformadora (RICHETTI; FILHO, 2014; ABRAHÃO; GODOY; HALPERN, 2013; NASCIMENTO et. al. 2011; SOUSA, et al., 2010; SANTOS, 2008; SANTOS; DIAS; MARTINS, 1995). Nesse sentido, é preciso que a inserção de temas como o uso racional e a propaganda de medicamentos no currículo das séries iniciais deve ser baseada em aspectos que enfoquem a prevenção e o uso indevido de medicamentos (BRASIL, 2009, 2007).

\section{Considerações Finais}

Os artigos que invisibilizam a escola como um local para se adquirir informações sobre o uso dos medicamentos, estão em consonância com os discursos do setor farmacêutico, representando a lógica do consumo e posturas que vão de encontro com o preceito principal da saúde coletiva: prevenção e promoção de saúde para todos.

\footnotetext{
${ }^{5}$ Lógica mercadológica "[...] que consiste em induzir o consumo de medicamentos [...]" (CORRER; OTUKI, 2013, p. 8).
} 
O material categorizado que demonstra a importância da escola como um espaço fundamental de disseminação de conhecimentos sobre o uso de medicamentos apresenta um discurso de prevenção, interdisciplinar, pautado na medicina social e em consonância com o Movimento de Reforma Sanitária. Estes artigos apresentam uma relação mais estreita com a escola e com a capacidade que a mesma tem de promover saúde. Essa prática pode ocorrer de forma intersetorial, apresentando e fomentando a construção de espaços conjuntos dos profissionais da saúde e da educação. Assim, professores e comunidade escolar serão capazes de desenvolver, por meio de práticas pedagógicas críticas, ações que podem possibilitar a discussão do tema do uso de medicamentos.

Existe a necessidade de uma prática do uso racional de medicamentos nas escolas e esta ação se constituirá em ferramenta de promoção da saúde. Essa abordagem faz parte de uma nova prática social que fortalece a relação professor/escola/profissional de saúde.

\section{Referências}

ABJAUDE, S. A. R. et al. Promoção da saúde: orientação para alunos do Ensino Fundamental. Conexão UEPG, v. 8, n. 2, 2012.

ABRAHÃO, R. C.; GODOY, J. A.; HALPERN, R. Automedicação e comportamento entre adolescentes em uma cidade do Rio Grande do Sul. Aletheia, 41, p. 134-153, maio/ago. 2013.

ALMEIDA, C. et al. Levantamento do uso de medicamentos por estudantes do Ensino Médio em duas escolas de Porto Alegre, RS, Brasil. Revista Ciência \& Educação, v. 18, n. 1, p. 215-239, 2012.

ANDRADE, R. M.; SOUSA, M. H. Automedicação como ferramenta para o ensino de química no Ensino Médio. Enciclopédia Biosfera, Goiânia: Centro Científico Conhecer, v. 9 , n. 17, p. 3.001, 2013.

ANGELL, M. A verdade sobre os laboratórios farmacêuticos. Rio de Janeiro: Ed. Record, 2009.

AQUINO, D. S. Por que o uso racional de medicamentos deve ser uma prioridade? Ciência \& Saúde Coletiva, 13 (Sup.): 733-736, 2008.

BARROS, J. A. C. A atuação dos balconistas de farmácia - ajudando a promover o uso racional de medicamentos? Jornal Brasileiro de Medicina, n. 73 (2):120-127, 1997. 
BARTA, R. L.; OLIVEIRA, K. R. A prática da automedicação por funcionários de uma instituição de Ensino Superior portadores de enxaqueca. Revista de Ciências Farmacêuticas Básica e Aplicada, 31(2): 183-191, 2010.

BOCHNER, R. Perfil das intoxicações em adolescentes no Brasil no período de 1999 a 2001. Cad. Saúde Pública, Rio de Janeiro, 22(3):587-595, mar. 2006.

BRASIL. Parâmetros Curriculares Nacionais: saúde. Brasília, 1997. Disponível em: <http://portal.mec.gov.br/seb/arquivos/pdf/saude.pdf>. Acesso em: 18 maio 2013.

. Diretrizes Curriculares Nacionais para os Cursos de Ciências Biológicas. Brasília, DF: Ministério da Educação; Conselho Nacional de Educação, 2001.

. Política Nacional de Promoção da Saúde. Brasília, DF: Ministério da Saúde, 2006.

Agência Nacional de Vigilância Sanitária. Gerência de Monitoramento e Fiscalização de Propaganda, de Publicidade, de Promoção e de Informação de Produtos Sujeitos à Vigilância Sanitária. Projeto educação e promoção da saúde no contexto escolar: o contributo da Agência Nacional de Vigilância Sanitária para o uso racional de medicamentos. Caderno do Professor. Brasília: Anvisa, 2007.

. Saúde na escola. Cadernos de Atenção Básica n. 24; Série B. Textos Básicos de Saúde. Brasília, DF: Ministério da Saúde; Secretaria de Atenção à Saúde; Departamento de Atenção Básica, 2009.

. Diretrizes Curriculares Nacionais Gerais da Educação Básica. Brasília, DF: Ministério da Educação; Secretaria de Educação Básica, 2013a.

. Sistema Nacional de Informações Tóxico-Farmacológicas, 2013b. Disponível em: <http://sinitox.icict.fiocruz.br/>. Acesso em: 3 maio 2017.

- Ministério da Saúde. Cartilha para a promoção do uso racional de medicamentos. Brasília: Ministérios da Saúde, 2015.

CÂMARA, A. M. C. S. et al. Percepção do processo saúde-doença: significados e valores da educação em saúde. Revista Brasileira de Educação Médica, 36 (1, Supl. 1): 40-50, 2012.

CARDOSO, V.; REIS, A. P.; IERVOLINO, S. A. Escolas promotoras de saúde. Revista Bras. Crescimento Desenvolv. Hum., 18(2): 107-115, 2008.

CARMO-JÚNIOR, N. M. A indústria farmacêutica e sua contribuição ao uso (ir)racional de medicamentos: o domínio mercadológico em detrimento das ações de saúde. 2013. 72f. (Monografia) -Universidade Federal do Maranhão, São Luís, MA, 2013.

CARVALHO, S. R. et al. Medicalização: uma crítica (im) pertinente? Physis Revista de Saúde Coletiva, Rio de Janeiro, 25 (4): 1.251-1.269, 2015. 
CASEMIRO, J. P.; FONSECA, A. B. C.; SECCO, F. V. M. Promover saúde na escola: reflexões a partir de uma visão sobre saúde escolar na América Latina. Ciência \& Saúde Coletiva, 19(3):829-840, 2014.

CATALÁN, V. G. El profesorado ante la educación y promoción de la salud en la escuela. Didácticas de Las Ciências Experimentales y Sociales, n. 23, p. 171-180, 2009.

CORRÊA, A. D. et al. Uma abordagem sobre o uso de medicamentos nos livros didáticos de biologia como estratégia de promoção de saúde. Ciência \& Saúde Coletiva, 18(10): 3.071-3.081, 2013.

CORRER, C. J.; OTUKI, M. F. (Org.). A prática na farmácia comunitária. Porto Alegre: Artmed, 2013.

COSTA. M. V.; SILVEIRA, R. H.; SOMMER, L. H. Estudos culturais em educação e pedagogia. Revista Brasileira de Educação, n. 23, maio/jul./ago. 2003.

COSTA, A. C. et al. A automedicação como tema gerador para o ensino de ciências - um enfoque no ensino de química. In: CONGRESSO NORTE-NORDESTE DE QUÍMICA, 4., 2011.Anais... Disponível em: <www.annq.org/congresso2011/arquivos/1300408099. pdf>. Acesso em: 5 nov. 2016.

COSTA, G. M. C. et al. Promoção de saúde nas escolas na perspectiva de professores do ensino fundamental. Rev. Eletr. Enf. [Internet], 15(2): 506-15, abr./jun., 2013.

COSTA, F. S.; SILVA, J. L. L.; DINIZ, M. I. G. A importância da interface educação/ saúde no ambiente escolar como prática de promoção da saúde. Informe-se em promoção da saúde, v. 4, n. 2, p. 30-33, 2008.

DAL PIZZOL, T. S. et al. Uso não-médico de medicamentos psicoativos entre escolares do Ensino Fundamental e Médio no Sul do Brasil. Cad. Saúde Pública, Rio de Janeiro, 22(1):109-115, jan. 2006.

DANDOLINI, B. W. et al. Uso racional de antibióticos: uma experiência para educação em saúde com escolares. Ciência e Saúde Coletiva, v. 17, n. 5, 2012.

DISTRITO FEDERAL. Secretaria de Estado de Educação. Subsecretaria de Educação Básica. Currículo em Movimento da Educação Básica. Ensino Fundamental: anos iniciais. 2014.

FERRARI DE LIMA, D.; MALACAME, V.; STRIEDER, D. M. O papel da escola na promoção da saúde - uma mediação necessária. EccoS Revista Científica, n. 28, maio/ ago. 2012.

FOELLMER, L.; OLIVEIRA, K. R.; MOREIRA, A. C. Uso racional de medicamentos: prioridade para a promoção da saúde. Revista Contexto \& Saúde, Ijuí: Ed. Unijuí, v. 9, n. 18, jan./jun. p. 53-62, 2010. 
FOUCAULT, M. Crise da medicina ou crise da antimedicina. Verve, 18: 167-194, 2010.

GOMES, C. D.; HORTA, N. C. Promoção da saúde do adolescente em âmbito escolar. Revista APS, Juiz de Fora, v. 13, n. 4, p. 486-499, out./dez. 2010.

GONÇALVES, F. D. et al. Health promotion in primary school. Interface - Comunic., Saúde, Educ., v. 12, n. 24, p. 181-92, jan./mar. 2008.

KOVACS, F. T.; BRITO, M. F. M. Percepção da doença e automedicação em pacientes com escabiose. An. Bras. Dermatol., 81(4):335-340, 2006.

LAGO, A. S. et al. Proposta pedagógica: uma discussão sobre o tema da automedicação nas aulas de química no Ensino Médio. 2016, 37 f. Trabalho (Conclusão de Curso de Química) - Universidade Estadual do Maranhão, Governador Nunes Freire, Programa Darcy Ribeiro, 2016.

LAURELL, A. C. La salud-enfermedad como proceso social. Revista Latinoamericana de Salud, México, 2, p. 7-25, 1982.

LEITE, S. N.; VIEIRA, M.; VEBER, A. P. Estudos de utilização de medicamentos: uma síntese de artigos publicados no Brasil e América Latina. Ciências e Saúde Coletiva, 13, (Sup):793-802, 2008.

MACHADO, L. V.; LESSA, P. S. Medicalização da vida: ética, saúde pública e indústria farmacêutica. Psicologia \& Sociedade, 24(3), 741-743, 2012.

MANGUEIRA, V. M. et al. Influência da atividade educativa sobre o uso racional de medicamentos para professores do ensino infantil da Creche-Escola do Centro de Educação da Universidade Federal da Paraíba. Centro de Ciências da Saúde, Departamento de Fisiologia e Patologia, Universidade Federal da Paraíba, 2011. p. 2. Disponível em: $<$ www.prac.ufpb.br/anais/XIIIENEX_XIVENID/.../6CCSDFPPX10-P.doc>. Acesso em: 5 jun. 2014.

MINAYO, M. C. S. O desafio do conhecimento: pesquisa qualitativa em saúde. 13. ed. São Paulo: Hucitec, 2013.

MOTA, D. F. S. Trabalhar a educação para a saúde nas escolas: percepção de profissionais de saúde e de professores. 2011. Dissertação (Mestrado em Educação para a Saúde) - Universidade do Porto, Porto, 2011.

NASCIMENTO, M. C. Medicamentos, comunicação e cultura. Ciência \& Saúde Coletiva, 10 (supl): 179-193, 2005.

NASCIMENTO, T. C. S. et al. Trabalho educativo com estudantes de Ensino Fundamental: uma abordagem sobre medicamentos, alimentação e legislação de propaganda. Educação e Informação em Saúde: caderno de textos acadêmicos, p.56-67, 2011. 
OLIVA, R. Uso racional de medicamentos: uma responsabilidade de todos. Fundação para o remédio popular, 2007. Disponível em: <http://ses.sp.bvs.br/local/File/Ricardo\%20Oliva_uso\%20racional\%20de\%20medicamentos.pdf $>$. Acesso em: 8 mai. 2017.

ORGANIZAÇÃO MUNDIAL DE SAÚDE (OMS). Medicines: rational use of medicines. Fact sheet, n. 338, may 2010. Disponível em: <http://www.wiredhealthresources.net/ resources/NA/WHO-FS_MedicinesRationalUse.pdf>. Acesso em: 9 mai. 2017.

Perspectivas políticas sobre medicamentos de la OMS. Promoción del uso racional de medicamento: componentes centrales. Washington, 2002. Disponível em: <http://apps.who.int/medicinedocs/pdf/s4874s/s4874s.pdf>. Acesso em: 8 mai. 2017.

ORGANIZAÇÃO PAN-AMERICANA DA SAÚDE (Opas). Ministério da Saúde; Agência Nacional de Vigilância Sanitária. Relatório de Oficina de Trabalho. Uso racional de medicamentos na perspectiva multiprofissional. Brasília, DF: Organização Pan-Americana da Saúde, 2007.

PALACIOS, M.; REGO, S.; LINO, M. H. Promoção e propaganda de medicamentos em ambientes de ensino: elementos para o debate. Interface: Comunicação, Saúde e Educação, v. 12, n. 27, p. 893-905, out./dez. 2008.

RICHETTI, G. P.; FILHO, J. P. A. Automedicação no ensino de química: uma proposta interdisciplinar para o Ensino Médio. Educação Química, 25(E1), 2003-2009, 2014.

SANTOS, M. G.; DIAS, A. G. P.; MARTINS, M. M. Conhecimento e uso da medicina alternativa entre alunos e professores de primeiro grau. Revista de Saúde Pública, 29 (3), 221-7, 1995.

SANTOS, K. F.; BÓGUS, C. M. A percepção de educadores sobre a escola promotora de saúde: um estudo de caso. Rev. Bras. Crescimento Desenvolv. Hum., 17(3): 123-133, 2007.

SANTOS, D. S. F. A. V. Uso racional de medicamentos e formação de professores. 2008. 72f. Dissertação (Mestrado em Educação) - Universidade de Uberaba, Programa de Mestrado em Educação, Uberaba, 2008.

SÁ-SILVA, J. R.; ALMEIDA, C. D.; GUINDANI, J. F. Pesquisa documental: pistas teóricas e metodológicas. Revista Brasileira de História e Ciências Sociais, ano I, n. I, jul. 2009.

SIGNOR, R.C.F.; BERBERIAN, A.P.; SANTANA, A.P. A medicalização da educação: implicações para a constituição do sujeito aprendiz. Educ. Pesqui., São Paulo: Ahead of print, out. 2016.

SILVA, C. H.; GIUGLIANE, E. R. J. Consumo de medicamentos em adolescentes escolares: uma preocupação. Jornal de Pediatria, 80 (4): 326-32, 2004. 
SILVA, M. V. S. et al. Consumo de medicamentos por estudantes adolescentes de escola de Ensino Fundamental do município de Vitória. Revista de Ciências Farmacêuticas Básica e Aplicada, 30 (1):99-104, 2009.

SILVA, I. M. et al. Automedicação na adolescência: um desafio para a educação em saúde. Ciência \& Saúde Coletiva, 16(Supl. 1): 1.651-1.660, 2011.

SILVA, M. L. M.; PINHEIRO, P. C. Educação Química e o problema da automedicação: relato de sala de aula. Química Nova Escola, v. 35, n. 2, p. 92-99, maio 2013.

SOUSA, I. F. et al. Uso racional de medicamentos: relato de experiência no Ensino Médio da Unesc, Rev. Bras. Educ. Med., Criciúma, SC. vol. 34, n. 3, jul./set. 2010.

VITOR, R. S. et al. Padrão de consumo de medicamentos sem prescrição médica na cidade de Porto Alegre, RS. Ciência e Saúde Coletiva, 13:737-43, 2008.

ZANCUL, M. S.; COSTA, S. S. Concepções de professores de Ciências e de Biologia a respeito da temática educação em saúde na escola. Experiências em Ensino de Ciências, v. 7, n. 2, 2012.

ZANCUL, M. S.; GOMES, P. H. M. A formação de licenciandos em Ciências Biológicas para trabalhar temas de educação em saúde na escola. Ensino, Saúde e Ambiente, v. 4, p. 49-61, 2011. 
Anexo - Publicações científicas e suas respectivas categorias conforme análise

\begin{tabular}{|c|c|c|c|}
\hline Categorias & Documento/Artigo & $\begin{array}{c}\text { Ano de } \\
\text { publicação }\end{array}$ & Autor \\
\hline \multirow{15}{*}{$\begin{array}{l}\text { Publicações científicas que } \\
\text { invisibilizam a escola na } \\
\text { discussão sobre o uso ra- } \\
\text { cional de medicamentos }\end{array}$} & $\begin{array}{l}\text { Discutindo, no cotidiano escolar, } \\
\text { o papel da educação em saúde, } \\
\text { em relação ao uso indevido de } \\
\text { medicamentos por adolescentes. }\end{array}$ & 2007 & $\begin{array}{l}\text { Funghetto; } \\
\text { Pereira }\end{array}$ \\
\hline & $\begin{array}{l}\text { Parâmetros Curriculares Nacio- } \\
\text { nais - Tema transversal saúde }\end{array}$ & 1997 & Brasil \\
\hline & $\begin{array}{l}\text { A atuação dos balconistas de } \\
\text { farmácia - Ajudando a promover } \\
\text { o uso racional de medicamentos? }\end{array}$ & 1997 & Barros \\
\hline & $\begin{array}{l}\text { Consumo de medicamentos em } \\
\text { adolescentes escolares: uma } \\
\text { preocupação. }\end{array}$ & 2004 & $\begin{array}{c}\text { Silva; } \\
\text { Giugliane }\end{array}$ \\
\hline & $\begin{array}{l}\text { Percepção da doença e auto- } \\
\text { medicação em pacientes com } \\
\text { escabiose }\end{array}$ & 2006 & Kovacs \\
\hline & $\begin{array}{l}\text { Uso não médico de medicamen- } \\
\text { tos psicoativos entre escolares do } \\
\text { Ensino Fundamental e Médio no } \\
\text { Sul do Brasil. }\end{array}$ & 2006 & Dal Pizzol \\
\hline & $\begin{array}{l}\text { Uso racional de medicamentos } \\
\text { na perspectiva multiprofissional }\end{array}$ & 2007 & Opas \\
\hline & $\begin{array}{l}\text { Uso racional de medicamentos: } \\
\text { uma responsabilidade de todos. }\end{array}$ & 2007 & Oliva \\
\hline & $\begin{array}{l}\text { Estudos de utilização de medica- } \\
\text { mentos: uma síntese de artigos } \\
\text { publicados no Brasil e América } \\
\text { Latina }\end{array}$ & 2008 & Leite et al. \\
\hline & $\begin{array}{l}\text { Promoção e propaganda de me- } \\
\text { dicamentos em ambientes de } \\
\text { ensino: elementos para o debate. }\end{array}$ & 2008 & $\begin{array}{l}\text { Palácios } \\
\text { et al. }\end{array}$ \\
\hline & Saúde na escola & 2009 & Brasil \\
\hline & $\begin{array}{l}\text { Uso racional de medicamentos: } \\
\text { relato de Experiência no Ensino } \\
\text { Médico da Unesc, Criciúma/SC }\end{array}$ & 2009 & $\begin{array}{l}\text { Sousa et } \\
\text { al. }\end{array}$ \\
\hline & $\begin{array}{l}\text { Consumo de medicamentos por } \\
\text { estudantes adolescentes de Es- } \\
\text { cola de Ensino Fundamental do } \\
\text { município de Vitória }\end{array}$ & 2009 & Silva et al. \\
\hline & $\begin{array}{l}\text { Educação e informação em saúde: } \\
\text { caderno de textos acadêmicos }\end{array}$ & 2011 & Brasil \\
\hline & $\begin{array}{l}\text { Levantamento do uso de medica- } \\
\text { mentos por estudantes do Ensino } \\
\text { Médio em duas escolas de Porto } \\
\text { Alegre, RS, Brasil }\end{array}$ & 2012 & $\begin{array}{l}\text { Almeida } \\
\text { et al. }\end{array}$ \\
\hline
\end{tabular}




\begin{tabular}{|c|c|c|c|}
\hline \multirow{8}{*}{$\begin{array}{l}\text { Publicações científicas que } \\
\text { visibilizam a escola na dis- } \\
\text { cussão sobre o uso racional } \\
\text { de medicamentos }\end{array}$} & $\begin{array}{l}\text { Conhecimento e uso da medicina } \\
\text { alternativa entre alunos e profes- } \\
\text { sores de primeiro grau. }\end{array}$ & 1995 & Santos et al \\
\hline & $\begin{array}{l}\text { Currículo da educação básica } \\
\text { das escolas públicas do Distrito } \\
\text { Federal: anos iniciais } \\
\end{array}$ & 2014 & $\begin{array}{l}\text { Distrito Fe- } \\
\text { deral }\end{array}$ \\
\hline & $\begin{array}{l}\text { Projeto educação e promoção } \\
\text { da saúde no contexto escolar: o } \\
\text { contributo da Agência Nacional } \\
\text { de Vigilância Sanitária para o uso } \\
\text { racional de medicamentos }\end{array}$ & 2007 & Brasil \\
\hline & $\begin{array}{l}\text { Uso racional de medicamentos e } \\
\text { formação de professores }\end{array}$ & 2008 & Santos \\
\hline & $\begin{array}{l}\text { Promoção da saúde: orientação } \\
\text { para alunos do Ensino Funda- } \\
\text { mental }\end{array}$ & 2012 & $\begin{array}{l}\text { A bja ude } \\
\text { et al. }\end{array}$ \\
\hline & $\begin{array}{l}\text { Influência da atividade educativa } \\
\text { sobre o uso racional de medi- } \\
\text { camentos para professores do } \\
\text { ensino infantil da Creche-Escola } \\
\text { do Centro de Educação da Uni- } \\
\text { versidade Federal da Paraíba }\end{array}$ & 2011 & $\begin{array}{l}\text { Mangueira, } \\
\text { et al. }\end{array}$ \\
\hline & $\begin{array}{l}\text { Uso Racional de Antibióticos: } \\
\text { uma experiência para educação } \\
\text { em saúde com escolares. }\end{array}$ & 2012 & $\begin{array}{l}\text { Dandolini } \\
\text { et al. }\end{array}$ \\
\hline & $\begin{array}{l}\text { Uma abordagem sobre o uso de } \\
\text { medicamentos nos livros didáti- } \\
\text { cos de biologia como estratégia } \\
\text { de promoção da saúde. }\end{array}$ & 2013 & $\begin{array}{l}\text { Correa et } \\
\text { al. }\end{array}$ \\
\hline
\end{tabular}

Recebido em: 13/9/2016

Aceito em: 14/6/2017 